\title{
Linx
}

Revue des linguistes de l'université Paris X Nanterre

$75 \mid 2017$

Imaginaires de la ponctuation. Ordre et inquiétude du discours

\section{Une ponctuation fantasmée (hantée par l'oral) : des signes imparfaits aux signes rêvés ...}

\section{Myriam Ponge}

\section{(2) OpenEdition}

\section{Journals}

Édition électronique

URL : http://journals.openedition.org/linx/1953

DOI : $10.4000 / \operatorname{linx} .1953$

ISSN : 2118-9692

Éditeur

Presses universitaires de Paris Nanterre

Édition imprimée

Date de publication : 22 décembre 2017

Pagination : 199-224

ISBN : 978-2-84016-308-4

ISSN : 0246-8743

Référence électronique

Myriam Ponge, «Une ponctuation fantasmée (hantée par l'oral) : des signes imparfaits aux signes rêvés ... », Linx [En ligne], 75 | 2017, mis en ligne le 23 novembre 2018, consulté le 19 avril 2019. URL: http://journals.openedition.org/linx/1953 ; DOI : 10.4000/linx.1953

Ce document a été généré automatiquement le 19 avril 2019

Département de Sciences du langage, Université Paris Ouest 


\title{
Une ponctuation fantasmée (hantée par l'oral) : des signes imparfaits aux signes rêvés ...
}

\author{
Myriam Ponge
}

1 Présentée traditionnellement comme «système de renfort de l'écriture » (Catach, 1991 : 49) ${ }^{1}$, la ponctuation - qui s'est enrichie de nombreux signes (depuis la triade grecque originelle) -n'en est pas moins perçue, par bien des scripteurs, comme un système qui demeure " perfectible ». Au carrefour de l'orthographe et de la typographie, cet objet aux contours flous (renvoyant à des éléments et des niveaux d'analyse variables - du mot à la page $^{2}-$ ), pourrait s'apparenter à un système " ouvert », susceptible de fluctuer au gré de l'évolution des pratiques d'écriture et de lecture.

2 Par ses caractéristiques, la ponctuation ne saurait être régie par des normes trop strictes, laissant entrevoir la part de jeu linguistique inhérente à l'appropriation d'un système cette marge de liberté dont peut se prévaloir le scripteur par ses choix stylistiques. Spécialistes et profanes la commentent ; elle peut susciter des débats passionnés (révélant les fascinations ou rejets exercés par certains signes), inspirer des innovations (par un usage particulier du système, la création de nouveaux ponctuants), etc. - et générer autant d'attitudes linguistiques qui témoignent de la dimension affective associée à cet objet singulier ${ }^{3}$. La ponctuation s'avère ainsi être un lieu privilégié d'investissement de l' imaginaire linguistique (Houdebine, 2002) ${ }^{4}$. A partir d'une analyse des critiques traditionnelles adressées à la ponctuation et de l'examen de diverses propositions d'ajout de signes, nous entendons questionner les représentations dominantes qui façonnent cet imaginaire. Il s'agira notamment d'observer dans quelle mesure les discours et pratiques innovantes contemporaines pourraient rester tributaires d'une conception oralisante.

Même si le système « idéal » de ponctuation qui se dessine en creux (à partir des critiques et des propositions de "perfectionnement») ne saurait être le même pour tous, l'étude approfondie de ses éléments constitutifs permettra de révéler les axes principaux autour desquels se structurent les attentes et les représentations fondamentales associées à la ponctuation. Ainsi, au-delà de l'apparente disparité des propositions (qui ne fait que 
réaffirmer la dimension polyfonctionnelle des signes du système), seront distinguées les conceptions majeures associées à un "perfectionnement " de la ponctuation qui oscille entre quête d'un système idéal de notation de la voix et exploration des potentialités figuratives d'une écriture idéogrammatique, voire pictogrammatique.

Il ne s'agira pas ici de se prononcer sur la recevabilité ou le bien-fondé linguistique des critiques à l'égard de la ponctuation ou des voies de "perfectionnement » proposées, mais de rendre compte de l'existence de tels discours, d'en analyser les caractéristiques essentielles, afin de révéler l'imaginaire linguistique dont ils sont porteurs.

\section{La ponctuation comme voie de perfectionnement de l'écriture}

\subsection{Le lieu commun de la ponctuation imparfaite}

5 Dans son Essai sur l'origine des langues, Rousseau consacre un chapitre à l'écriture où il déplore la perte de "vivacité » de la langue. Selon lui, seul un perfectionnement de la ponctuation pourrait éviter la tendance à l'alourdissement de la forme écrite : "Le meilleur de ces moyens et qui n'auroit pas ce défaut, seroit la ponctuation, si on l'eût laissée moins imparfaite » (1968 [1781] : 69)5.

6 Au-delà de Rousseau, nombreux sont les auteurs - de diverses langues ${ }^{6}$ - qui déplorent l'insuffisance des moyens de ponctuation dont ils disposent, insistant à tel point sur la nécessité de l'enrichir d'autres signes que cette pétition a pu être qualifiée de " lamentación tópica " par l'Espagnol Polo (1990: 49). Critiquée, qualifiée de "pauvre», « insuffisante », " ambiguë» voire "vicieuse », la ponctuation imparfaite apparaît bien comme un des lieux communs les mieux partagés, à partir duquel se cristallisent espoirs et attentes des locuteurs-scripteurs.

7 Dénoncer la "pauvreté ${ }^{7}$ de la ponctuation au regard de la supposée "richesse » de la langue (dans sa manifestation orale), s'appuyer sur les ambiguïtés qui peuvent alors résulter de l'emploi de signes nécessairement limités (cas d'homographie et de synonymie, selon Demanuelli, 1987 : 20) sont autant d'arguments récurrents brandis par ceux qui aspirent à un perfectionnement de la ponctuation.

8 Transparaissent ici, derrière ces critiques, des représentations plus profondes associées à la nostalgie de la "parole vive » et à la défiance générale qu'inspire l'écrit - et qui se décline de diverses manières, comme l'a montré Derrida (1967) en dénonçant un « phonocentrisme » dominant. Ainsi, plus que la ponctuation, c'est le système d'écriture dans son ensemble qui est mis en cause, son incapacité à rendre compte fidèlement de la langue, dans ses nuances et ses richesses - ce que Butor (1964: 393) évoque comme «les insuffisances de l'écriture pour la transcription de ce que l'oreille entend ».

Critiquée - y compris très sévèrement par certains auteurs (Fourier, infra) -, la ponctuation n'en est pas moins perçue comme un levier de perfectionnement de l'écriture; elle se voit dès lors dotée, dans sa forme idéalisée, des moyens qui permettraient de remédier aux insuffisances de l'écrit. En dénonçant ses imperfections, les auteurs réaffirment leur attachement à la ponctuation et leur croyance aux vertus singulières d'un système de renfort de l'écriture. Se dessinent ainsi, en creux, les principales fonctions qui lui sont attribuées, tant au niveau logico-syntaxique, pour son rôle diacritique d'aide à la clarification du discours, qu'au niveau prosodique, voire 
expressif. A cet égard, semble prévaloir la référence implicite à un système idéal de transcription qui permettrait de transmettre un discours oral dans les moindres de ses nuances ; parmi les critiques, évoquer la "pauvreté » de la ponctuation revient bien souvent à faire état du piètre système de notation dont dispose l'écrit pour tenter de rendre compte d'un message oral et de son contexte d'énonciation, riche d'informations suprasegmentales et paralinguistiques.

Ainsi, bien qu'imparfaite, la ponctuation - compte tenu de sa spécificité au sein de l'écrit - apparaît comme étant la plus à même d'établir des passerelles vers le niveau suprasegmental, cette "troisième articulation du langage" qu'évoque Catach (en référence à l'expression " des pauses, du rythme, de la ligne mélodique, de l'intonation " [1980:17]). Ses qualités en font ainsi un domaine à part, qui inspire les scripteurs soucieux, à travers les siècles, de trouver des moyens d'améliorer la notation de la langue. Sand (1873 [1871]: 99) ne considérait-elle pas déjà la ponctuation comme «un perfectionnement du langage »? Avant d'aborder les propositions d'ajout de signes des $\mathrm{XX}^{\mathrm{e}}$ et $\mathrm{XXI}^{\mathrm{e}}$ siècles, examinons quelques-unes des solutions envisagées par ceux qui rêvaient déjà, dans des périodes antérieures, d'une ponctuation moins imparfaite; les expériences innovantes dans ce domaine ne sont pas le seul apanage de la modernité et de la post-modernité.

\subsection{Premières pistes d'amélioration de la ponctuation (XVI-XIXes)}

11 A partir des critiques précédemment évoquées, la nécessité de remédier aux « ambigüités » associées à la "pauvreté » et «l'imprécision » des signes de ponctuation apparait primordiale. Rousseau (1968 [1781]: 69) attire ainsi l'attention sur des cas d'énoncés ambigus, non discriminés par une ponctuation spécifique, pour défendre l'introduction d'un point vocatif:

[...] comment distinguer par écrit un homme qu'on nomme d'un homme qu'on appelle? C'est là une équivoque qu'eût levée le point vocatif. La même équivoque se trouve dans l'ironie quand l'accent ne la fait pas sentir.

La clarification du discours, avec la mise en évidence ici de la fonction discriminante de la ponctuation, reste corrélée à une conception oralisante de la langue (voir «l'accent») - la réflexion de Rousseau s'intégrant à une critique générale des modes de notation de la « vivacité » de la langue parlée. Il est aussi fait incidemment référence au cas de l'ironie et à la nécessité de son repérage (exprimée ailleurs dans des propositions concrètes d'ajout de signes, infra). Les remarques de Rousseau seraient à rapprocher de celles de Duclos dont il aurait pu s'inspirer (Derrida, 1967) - qui évoque dans ses textes l'importance des « inflexions oratoires » (dont « la colère, le mépris, l'ironie, etc. », sans marques écrites ${ }^{8}$ ).

Nombre d'exemples témoignent de la prégnance du modèle oral, mais il est des cas où l'aide à l'oralisation des textes (diction, déclamation) justifie une approche valorisant, de manière plus explicite, les fonctions prosodiques de la ponctuation.

Les propositions de Gérard de Vivre, à la fin du XVI ${ }^{e}$ siècle - à une époque où les règles de ponctuation sont en cours d'établissement (Lemaire, 1998) - sont à associer à la pratique théâtrale. Par l'ajout de sept signes de didascalie, l'auteur-pédagogue entend faciliter l'interprétation de ses jeunes comédiens, en indiquant plus précisément des temps de pause, des variations de débit, voire un déplacement sur scène (cf. figure 1, pour un récapitulatif des valeurs assignées à ces signes ${ }^{9}$ ). 


\section{Lafignification des fignes, defquelsi'vferay \\ en toutes mes Comedies.}

\section{$t$ Ce figne, fignifie vne paufe. \\ [ Ceftuy deux. \\ * Ceftuy trois, Chacune paufe vaur vne re- prife d'haleine. \\ 28. Vn pourmenement par tout le Theatre. \\ की Ceci fignifie parler bas. \\ "Ceci fignifie, de parler plus vifte que le refte. \\ - Cecy de parler plus lenternent que le refte.}

FiguRE 1 : Extrait de G. de Vivre, Trois comédies francoises, Anvers, G. Janssens, 1595

La fonction générale d'aide à la lecture à voix haute ${ }^{10}$ est souvent évoquée pour rendre compte d'améliorations souhaitées en matière de ponctuation.

Ainsi, dans Le Traité du récitatif, Sieur de Grimarest ${ }^{11}$ (1707: 47-48) plaide pour un enrichissement du système :

Il seroit à souhaiter que l'on eût encore admis dans notre Langue des Points de commandement; d'ironie, de mépris; d'emportement; d'amour, \& de haine; de joie, \& de douleur : la lecture en seroit beaucoup plus aisée \& l'on donneroit à sa prononciation le sens qu'un Auteur auroit mis dans son ouvrage: Au lieu qu'incertain de ce qui va suivre, un Lecteur manque souvent le ton nécessaire à l'expression.

Il se garde pourtant bien de tenter de créer de tels signes, craignant d'inspirer le ridicule (1707 : 48). Soucieux de tirer le meilleur parti de la ponctuation existante pour guider davantage le lecteur dans l'oralisation d'un texte, il préconise de modifier la position des points d'admiration et interrogant (1707: 52-53) :

il me paroît que la marque de l'admiration, ou de l'interrogation devroit précéder la phrase : parce que la période pouvant être longue, \& le Lecteur n'étant point averti qu'elle roûle toute sur l'interrogation, par exemple, il pourroit changer de ton: ce qui seroit un défaut dans la prononciation [...].

La langue espagnole dispose, depuis la seconde moitié du XVIII ${ }^{e}$ siècle ${ }^{12}$, de la spécificité d'un marquage double (signes ouvrant et fermant d'interrogation et d'exclamation).Ce mode de notation ne manque pas d'être évoqué comme une innovation enviable en français (au XIX s., voir Jobard [1842, infra], Sand [1873 : 97]).

Les passerelles traditionnellement établies entre ponctuation et notation des dimensions suprasegmentales (pauses et intonations) pourraient être illustrées encore de bien des manières. Pour ne signaler qu'un dernier exemple relatif à cette époque, mentionnons Rétif de la Bretonne, connu aussi pour ses «modulations typographiques » (Butor, 1964 : 102). Dans les notes du Pied de Fanchette (1776[1769] : 280), il aspire à un modèle de notation plus précis pour transcrire l'oral ("grasseyement », « tons » variés) et évoque ces signes :

On pourrait, ce me semble, inventer quatre nouvelles ponctuations, qui faciliteraient infiniment cette utile méthode : le point précipitatif, le ralentissant, l'indignatif, l'attendrissant. Quelle clarté ne répandraient-ils pas dans le discours! et 
surtout que de parenthèses ils remplaceraient dans nos comédies nouvelles, nos romans du jour et nos opéras-bouffons!... Fourier accompagnent une réflexion plus vaste sur la création d'une "grammaire générale - l'une des voies de réforme envisagées pour combattre la «cataracte intellectuelle $»^{13}$. La longue note sur la ponctuation évoque la " pauvreté » d'un système à sept signes < ,;: .!? ) > pour dénoncer, au-delà de son ambigüité, l'expression d'un «chaos» voire d'une «anarchie universelle en grammaire». Le projet de refonte du système (cf. infra, 1829 [2001]: note 1, 511) n'en reste pas moins vague; l'auteur s'appuierait certes sur une augmentation significative du nombre de signes et une distinction de leur valeur, mais ne donne pas le moindre indice concernant leurs significations principales, passées ou futures :

Outre l'alphabet des lettres, il faudra créer celui de la ponctuation, qui doit contenir même nombre de signes [...]. Notre langue est si pauvre en ce genre, qu'on est obligé d'employer, ou le point, ou les deux points ; ce qui cause confusion.

J'avais commencé un travail sur la gamme de ponctuation, je l'avais poussé à 25 signes, appuyés d'exemples dénotant le ridicule et l'ambigüité de nos signes actuels : j'ai perdu ce travail avant qu'il fût achevé et je ne l'ai pas recommencé depuis. Observons à ce sujet que le premier de nos signes, le plus bas, nommé virgule , doit être différencié au moins en quadruple forme, pour faire apprécier les différentes portées de la virgule, ses acceptions qui, variant à l'infini, sont exprimées confusément par un seul signe, c'est le comble du désordre. Il en est de même des autres signes, ils cumulent 3 ou 4 sens : la ponctuation civilisée est un vrai chaos, comme l'orthographe, qui varie dans chacune des imprimeries de Paris [...].

La perspective « logique » que l'on pressent ici pour défendre l'ajout de nouveaux signes, accompagne plus largement les discours des grammairiens et imprimeurs-typographes $\mathrm{du} \mathrm{XIX}^{\mathrm{e}}$ siècle. Visant à une standardisation des usages pour l'édition des textes, ces derniers se référent essentiellement aux critères syntaxiques et logiques, s'éloignant en cela de l'héritage d'une tradition rhétorique qui a longtemps prévalu (en référence à un découpage en " périodes mélodiques » ${ }^{14}$.

Toutefois l'entrée dans l'ère normative du $\mathrm{XIX}^{\mathrm{e}}$ siècle n'exclut pas l'émergence de propositions originales, comme celles du lithographe belge, Jobard ${ }^{15}$, qui consacre un chapitre aux « lacunes de la typographie » (1842:349-351, pour la ponctuation). Il entend " perfectionner le cassier de nos imprimeurs ", insistant sur l'économie du système :

un point d'ironie représenté par une petite flèche [...]; un point d'irritation, un point d'indignation, un point d'hésitation, tout cela exprimé avec la même flèche placée dans quatre positions différentes. Nous proposons un point de sympathie (), d' antipathie )(, un point d'affliction, un point de satisfaction; un signe pour la voix haute

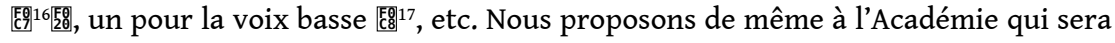
chargée de cette réforme, l'emploi d'un tiret pour le premier interlocuteur, de deux tirets [symboles à reproduire] pour le second, et de trois pour le troisième [...]. Nous ajouterons qu'il est de toute nécessité d'adopter l'usage espagnol, de placer points 
expressifs avant et après la phrase, de manière à indiquer au lecteur le sens de l'intonation qu'il doit prendre. Quatre signes nouveaux susceptibles de recevoir quatre positions différentes, ajoutés à la casse, suffiraient pour exprimer seize sentiments génériques dans lesquels viendraient se confondre leurs diverses nuances.

\section{d'habiter des propositions de perfectionnement de la ponctuation - entendue ici comme} système de notation des pauses et intonations.

\subsubsection{De nouveaux signes de pause}

D'après le Larousse de la Langue Française (1986: 4454), «tous les signes de ponctuation dénotent une pause, obligatoire ou facultative, signe d'une démarcation linguistique ou psychique [...] ». Des auteurs espagnols vont jusqu'à restreindre le champ de la ponctuation à cinq signes (point, virgule, point-virgule, deux-points, points de suspension) en fonction de ce critère ${ }^{20}$; quant à Damourette (1939), il distinguait « signes pausaux » (virgule, point, point-virgule) et «signes mélodiques ». Même si l'existence d'un rapport bi-univoque entre signes prosodique et graphique est contestable ${ }^{21}$, nombre 
d'auteurs restent attachés à cette correspondance et voient encore une manière de perfectionner le système en affinant le mode de notation des pauses.

31 Damourette (1939: 44) suggère de donner un signe spécifique à la "pausette ", en la notant par un point en haut ou une virgule inversée ${ }^{22}$. Parmi les écrivains, Claudel préconise l'espace blanc de "pause $»^{23}$ pour signaler un temps d'arrêt, de silence. La suppression de la ponctuation traditionnelle invite certains auteurs à s'appuyer sur la valeur des blancs typographiques; pour l'espagnol, Huidobro indique : «es más lógico cambiar la puntuación por blancos y espacios » (Tena, 2012).

A ces propositions s'ajoutent celles reposant sur l'introduction de signes musicaux: « soupirs ", « demi-soupirs » pour Güiraldes ${ }^{24}$; voire « signe de silence » pour Jouvet ${ }^{25}$.

Perfectionner la ponctuation pourrait ainsi consister à commencer par ajouter des signes de pauses, voire à s'inspirer au-delà du modèle d'écriture musicale (cf. 2.1.3).

\subsubsection{De nouveaux signes d'intonation...}

Les propositions visent ici à étoffer la catégorie des "signes d'intonation", traditionnellement circonscrite aux signes d'exclamation et d'interrogation ${ }^{26}$. Outre la logique des modalités d'énonciation, l'intonation a aussi un rôle expressif non négligeable relevant de la transmission de modalités d'énoncé (en lien avec l'attitude du locuteur); les points de suspension peuvent apparaître ainsi comme une marque complémentaire, combinable avec les précédents graphèmes intonatifs. La plupart des propositions s'intéresse à cette dernière dimension modale et vise à accroître les potentialités expressives des signes pour noter ces " variations d'ordre affectif " (liées à la définition de la ponctuation [TLFi]).

Dans Plumons l'oiseau, Bazin (1966: 141-143) suggère d'adopter six nouveaux points d'intonation : points d'acclamation, de doute, de certitude, d'autorité, d'ironie et d'amour (cf. pictogrammes, 3.2.1). Queneau avait déjà introduit le point d'indignation $<\mathrm{ii}>$ dans $L e$ Chiendent (1974 [1933] : 352). Le linguiste Nève (1998: 337-348) invite à compléter le système classique par des marques d'intonation inspirées de la langue des signes des sourds (en retenant certains des monèmes habituellement transmis par le visage et correspondant à des aspects prosodiques) : points d'ordre $\langle>>$, de doute $\langle\sim>$, d'humour $<\mu>$, d'optation-suggestion $\langle\wedge\rangle$, de crainte $\langle\mathrm{T}\rangle$, d'admiration $\langle *\rangle$, de joie $\langle\mathrm{U}\rangle$, de tristesse $\langle \#\rangle$, de nervosité $<>$ et d'ironie $<\$>$. L'instauration de ces signes permettrait de lever des ambiguïtés et de faciliter la lecture au premier « coup d'œil » $(1998: 347)$.

La nécessité du repérage d'une intention ironique à l'écrit est maintes fois évoquée, et à des époques fort variées : elle a donné lieu à l'invention de divers points d'ironie, depuis la forme la plus connue du signe d'Alcanter de Brahm $<$ ? (créé en 1899 et répertorié dans le Nouveau Larousse Illustré du début du XX $\mathrm{X}^{\mathrm{e}}$. $^{27}$; il serait aussi apparu en Espagne, sous la plume d'un dénommé Antonio Palmero.

Sans qu'ils s'accompagnent de propositions graphiques concrètes, nombre d'exemples témoignent encore de l'attrait qu'exerce un système apte à noter des intonations variées, liées à des états émotionnels spécifiques; pour l'espagnol, Borges (1996:262) reconnaitt qu'il aurait pu être intéressant de s'essayer à la création de nouveaux signes de ponctuation : «signos de indecisión, de conmiseración, de ternura, de valor psicológico o musical "; Martínez de Sousa (1996: 277) évoque l'économie verbale que représenterait la présence de signes pour noter : « el amor, la simpatía, el odio, el desprecio ». 

signes doubles de ponctuation espagnole est encore régulièrement envié28. Il aurait même été question d'intégrer ces signes à l'orthographe réformée du français ; d'après Védénina (1989: 22, n.1), « la table ronde CNRS, discutant les problèmes de l'orthographe française (janvier 1973), a jugé nécessaire cette manière d'annoncer d'avance au lecteur le sens modal d'une phrase ».

\subsection{Le modèle de l'écriture musicale}

Nostalgiques de cette "unité paradisiaque perdue $»^{29}$ où poésie et musique ne faisaient qu'un, nombre d'écrivains se réfèrent au modèle musical. Selon Adorno (2004: 42) : «Le langage n'est dans aucun autre de ses éléments aussi semblable à la musique que dans les signes de ponctuation ". Cette relation étroite peut être soulignée encore en référence à l'origine de certains signes de ponctuation, empruntés aux neumes (signes de pauses et intonation dans la musique liturgique [Catach, 1994 : 22-23 ; Parkes, 1993 : 305]).

Valéry aspirait ainsi à un système enrichi de nouveaux signes : «Pourquoi pas des signes comme en musique? (où il en manque aussi). / Signes de vitesse, de fortement articulé des arrêts de différentes durées. Des «vivace », «solenne », «staccato, scherzando.» (1989 [Cahiers I, 1894-1914] : 474).

Hormis les propositions ponctuelles d'ajout de signes existants inspirés de la musique ( supra, signes de pauses), certains auteurs invitent à faire de la page une véritable partition musicale. L'expérience mallarméenne constituant un modèle, comme le reconnaît Butor $(1964,398)$ : « Mallarmé estimait qu'il était temps que la littérature reprît à la musique son bien, et a essayé lui-même de faire un livre partition ». Par une spatialisation originale de la parole, accompagnée de procédés ponctuo-typographiques de mise en relief, peuvent être transposés les mouvements de la voix, la variation de son intensité, etc.

Dans «poésie courbe», Rebotier (2001) utilise divers modes de notation, mêlant diversement écriture spatialisante - accompagnée d'éléments de ponctuation classique et de signes directionnels - à des signes musicaux ${ }^{30}$. Les respirations sont indiquées par la mise en évidence, entre les groupes rythmiques, d'apostrophes <’> ; apparaissent des références à l'intensité (pianissimo a fortissimo), au tempo recommandé pour l'exécution orale du poème (prestissimo ou allegreto, par exemple, avec parfois la mention d'une cadence précise $[2001: 108,165])^{31}$. Güiraldes (1973: 31) aurait certainement été séduit par l'expérience d'une notation similaire dans ses poèmes - dont il soulignait la teneur vocale, en lien avec le chant.

43 Pour le théâtre, Feydeau, dans Occupe-toi d'Amélie (1995 [1908] : 218), s'inspire également de l'écriture musicale : "ne sachant pas comment donner l'intonation exacte de ces interjections, nous avons pris le parti de les noter musicalement »; sont disposées sur une portée, précédées d'un soupir, une croche et une noire pour transcrire l'interjection « Ehé $»^{32}$.

Si en littérature la référence au modèle musical est fréquente, les expériences qui en émanent sont variables : de la page-partition évoquée jusqu'au détournement de certains signes traditionnels de ponctuation, auxquels sont conférées des propriétés musicales (voir pour Mallarmé - d'après Wilker [2002: 24-25] - : l'usage d'un tiret de «suspens 
vibratoire " comparable à l'effet d'« un accord non résolu », en musique ; de points de suspension réduits à deux points, ou encore d'un deux-points comme signe de reprise).

\section{Vers une expressivité graphique renforcée: d'idéogrammes en pictogrammes}

La recherche de davantage d'expressivité - au-delà des tentatives de représentation des nuances intonatives évoquées -, peut conduire à des expériences variées, visant à explorer davantage les potentialités figuratives des signes et à en intégrer de nouveaux, iconiquement plus motivés ${ }^{33}$.

Ne pouvant prétendre à l'exhaustivité, nous tâcherons de dégager les mécanismes généraux qui président à la création de ces nouveaux signes.

\subsection{Idéogrammes de ponctuation}

47 Les signes de ponctuation expressive disposent de qualités idéogrammatiques indéniables, qui leur confèrent une autonomie sémantique telle qu'ils peuvent être utilisés seuls ou combinés, en dehors de tout contexte verbal; voir $<$ ? > pour signifier questionnement, doute, perplexité ; <!>, pour «étonnement », "surprise, indignation » (Anis, 1988) ; <...>, pour interruption, silence. D'après la typologie d'Anis (1988: 143), ces signes sont assimilables à des « logogrammes », issus de « topogrammes détournés ».

Parmi les créations dérivées, signalons d'abord l'interrobang ou exclarrogatif $<$ 嚂 $>^{34}$ (dû au publicitaire Speckter, en 1962) qui associe simultanément - et non plus successivement les signes expressifs d'interrogation et d'exclamation. Le procédé de composition peut s'appliquer à d'autres graphèmes : virgule et signe exclamatif pour la virgule d'exclamation (dotée, selon Dandoy $[2007,19]$ d'un « degré supplémentaire de finesse ») ${ }^{35}$; sur le même principe, la virgule interrogative; ou les virgules supensives - dites « comas suspensivas » en espagnol (Cabrera Infante [1976] <,,,',,'> utilise un nombre variable de virgules, selon l'effet souhaité : de la suspension jusqu'à l'expression d'un coma $^{36}$ ); en référence à l'espagnol, Dandoy (2007: 80) imagine encore la «coma de interrogación» (virgule d'interrogation de forme inversée). Bien d'autres créations s'appuyant sur des signes de ponctuation traditionnels sont encore envisageables; rappelons que Larbaud signalait à son époque les « deux points de suspension » de Fargue et le " virgule-tiret ».

49 Quelle que soit la fantaisie apparente de certaines propositions, il importe de noter qu'elles s'appuient sur la reconnaissance de la valeur sémantique des signes (indépendamment de toute référence à l'oral); elles se fondent ainsi sur le décryptage idéogrammatique de signifiés issus des ponctuants traditionnels.

\subsection{Une ponctuation pictogrammatique}

\subsubsection{Pictogrammes d'intonation expressive} pictogrammes ${ }^{37}$.

51 Les points d'intonation proposés par Bazin (supra, en lien avec la notation de l'oral) explorent les ressources figuratives de l'écrit. Le point d'acclamation est décrit ainsi : «Bras 
levés, c'est le $\mathrm{V}$ de victoire. C'est la représentation stylisée des deux petits drapeaux qui flottent au sommet de l'autobus, quand nous visite un chef d'Etat » (Bazin, 1966 : 142).

142

\section{PLUMONS L'OISEAU}

I) Le point d'amour: ?

Il est formé de deux points d'interrogation qui, en quelque sorte, se regardent et dessinent, au moins provisoirement, une sorte de coeur.

2) Le point de conviction: !

C'est un point d'exclamation transformé en croix.

3) Le point d'autorité: ?

Il est sur votre phrase, comme un parasol sur le sultan.

4) Le point d'ironie: *

C'est un arrangement de la lettre grecque $\psi$. Cette lettre (psi) qui représente une flèche dans l'arc, correspondait à ps: c'est-à-dire au son de cette même flèche dans l'air. Quoi de meilleur pour noter l'ironie ?

5) Le point d'acclamation: V

Bras levés, c'est le V de la victoire. C'est la représentation stylisée des deux petits drapeaux qui flottent au sommet de l'autobus, quand nous visite un chef d'État.

6) Le point de doute : ?

Il est comme vous : il hésite, il biaise, avant de tomber - de travers - sur son point.

Figure 2 : Bazin, Plumons l'oiseau (1966: 142)

Au-delà du champ littéraire, la ponctuation inspire aussi les graphistes. Nous rapportons ici deux expériences de nature pictogrammatique, développées à partir des signes de ponctuation.

La graphiste-designer Jane Secret propose une série de «signes d'intonation »: points d'humeur ("points d'ironie, d'autorité, de folie, de rêverie»), accents d'humeur ("le Surpris, l'Effrayé »), guillemets d'humeur («Les Énervés, Les Hautains, Les Obsédés, Les Solennels, Les Malheureux, Les Fatigués, Les Timides, Les Joyeux, Les Soucieux ») pour tenter de traduire les « tons » correspondants à ces divers états ${ }^{38}$.

Voici deux de ces points, pour apprécier leur motivation graphique :

\section{LE POINT
DE FOLIE ? \\ LES POINTS DE RÊVERIE .}

La dimension dynamique de la page web permet d'en renforcer l'expressivité.

Bien que la dimension visuelle soit ici fondamentale, la ponctuation n'en reste pas moins envisagée par son auteur en lien avec l'oral; le sous-titre retenu pour présenter l'ensemble des nouveaux signes l'indique : «extension orale du domaine de la ponctuation ». 
Par ailleurs, le jeune designer typographique, Thierry Fetiveau, défend dans sa rubrique typographie, l'introduction du caractère Andersen (idéal pour les contes), accompagné d'un "système de ponctuation complémentaire " de 15 éléments pour exprimer des émotions variées: joie, colère, exaspération, surprise, inquiétude, peur, empressement, consolation, tristesse, menace, solennité, moquerie, déception, plainte, amour ${ }^{39}$ :

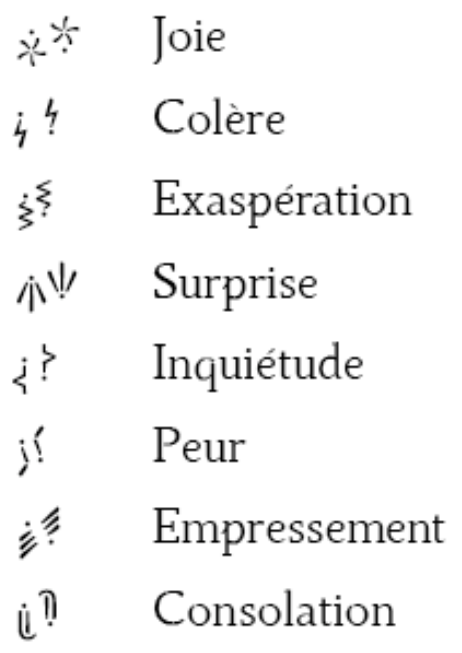

¿ ? Tristesse

$\dot{\Lambda} ! \quad$ Menace

$\dot{0}^{\mathrm{Q}} \quad$ Solennité

i) Moquerie

¿? Déception

$\doteq \overline{\overline{\bar{T}}} \quad$ Plainte

¿? Amour

\begin{tabular}{|c|c|c|c|}
\hline$\% \%$ & Happiness & $\dot{\varepsilon} ?$ & Sadness \\
\hline 34 & Anger & it & Threat \\
\hline$\xi_{\xi}$ & Exasperation & $\square_{\dot{0}}$ & Solaneity \\
\hline av & Surprise & i. & Moquery \\
\hline ? & Worry & $?_{j}$ & Disapoitement \\
\hline í! & Fear & $\doteqdot$ & Complaint \\
\hline 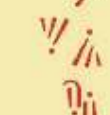 & $\begin{array}{l}\text { Hurry } \\
\text { Consolation }\end{array}$ & ¿s? & Love \\
\hline
\end{tabular}

Figures 3 et 4 : Illustrations extraites de la page web de Fetiveau consultée pour l'une, le 20-06-2014 et pour l'autre, le 28-01-2015

Outre la dimension pictogrammatique des signes, notons que cette proposition s'inspire de la ponctuation entourante de l'espagnol ; Fetiveau s'en explique ainsi : «Comme dans la ponctuation espagnole, ces symboles sont placés en début et fin de phrase afin de permettre au lecteur de lire dès le début avec le bon ton ». Est attribuée à la ponctuation une fonction clairement prosodique, d'aide à la lecture à voix haute. 


\subsubsection{Pictogrammes électroniques : émoticônes}

Résultant initialement de la combinaison de signes de ponctuation et de caractères spéciaux (ASCII), les pictogrammes contemporains - émoticônes (mot-valise : «étym. émotion + icône $»^{40}$ ) - méritent aussi d'être évoqués ; Anis les qualifie de "logogrammes pictographiques » $(1998: 212)$.

Désormais bien connus, ces nouveaux signes - qui ont considérablement évolué dans leur représentation (animée, multimodale) - permettent au scripteur de signifier «l'état d'esprit » (Clause, 2001: 79; Castells, 2001) dans lequel il se trouve lors de l'émission du message, en mimant graphiquement certains traits expressifs de son visage. Pour n'indiquer que quelques représentations élémentaires: expression de joie via <:-)> (transformée en ${ }^{\left[{ }_{4 A}^{4}\right.}{ }^{41}$ et variante japonaise $\left\langle\left({ }^{\wedge}{ }_{-}^{\wedge}\right)\right\rangle$ ) et son antonyme de tristesse <:-(>

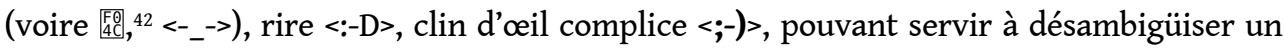
énoncé ironique. S'ajoutent bien d'autres variantes pour représenter attributs physiques ou autres caractéristiques personnalisées, ainsi qu'une gamme importante de pictogrammes d'origine japonaise, les emoji, qui viennent enrichir les messages électroniques. Les utilisateurs ${ }^{43}$ reconnaissent l'attractivité formelle de de ces nouveaux signes mais continuent de mettre en avant leur rôle dans la personnalisation d'un message pour transmettre des émotions et compenser l'absence d'un réel face à face ; la conversation orale non médiatisée sert bien encore de modèle de référence.

\subsubsection{Créativité et variété de pictogrammes}

L'exploration des voies figuratives, pour une ponctuation expressive, conduit à l'émergence de créations diverses qu'il serait difficile de recenser. Toutefois, à partir de l'échantillon de signes évoqués, un intérêt se dégage avant tout pour l'expression d'intonations et d'émotions (via diverses réinventions de signes d'ironie, de doute, de joie, de tristesse, d'autorité, d'amour). L'étendue de la palette de nuances émotives autorise de nombreuses possibilités d'invention - et autant d'hapax graphiques.

Compte tenu de la part subjective qui intervient dans les choix de représentations symboliques ou figuratives, des divergences formelles peuvent apparaitre entre les pictogrammes retenus pour exprimer portant une idée similaire. Les cas d'homographie ne sont pas non plus à exclure : le point d'ironie proposé par Secret

\section{LE POINT ?
[D'IRONIE ? ]}

ressemble au point de doute de Bazin

\section{[e point de doute : ?}


La poésie dite visuelle et les arts graphiques explorent le champ de la ponctuation, mais dans une toute autre perspective (si de nouvelles formes apparaissent, il ne s'agit pas pour autant d'en revendiquer l'usage dans un système de ponctuation améliorée) ; le seul esthétisme du signifiant graphique suffit à justifier la démarche créative (voir, par exemple, Blaine (1980) où chaque page est consacrée à un signe de ponctuation, existant ou fantasmé, jusqu'au "point de poésie "; ou encore les expériences de typoésie de Peignot (1993) - dont l'une joue à questionner le point d'ironie d'Alcanter de Brahm) :

\section{??}

Figure 5 : «Que n'a-t-on à cœur de réhabiliter le point d'ironie ? », Peignot (1993 : 162)

Entre poésie et recherche typographique, l'ouvrage de Dandoy (2007) part explicitement en quête des prochains nouveaux signes de ponctuation ${ }^{44}$. Parmi de multiples suggestions, apparaissent des séries de signes formellement motivés, dérivés de ponctuants, comme la « virgule flottante », les « points pensifs » (vs suspensifs), divers tirets (dont les « pensifs », « régressifs »), etc.

L'écriture pictogrammatique laisse ainsi la voie ouverte à la créativité idiolectale, faisant émerger diverses propositions (plus ou moins fantaisistes), guidées par la recherche d'une expressivité renforcée.

\subsection{Des idéogrammes de ponctuation au service d'une écriture universelle}

68 Associés à la recherche d'un langage universel, certains signes de ponctuation peuvent enfin être envisagés comme des idéogrammes privilégiés. L'intercompréhension visuelle prime ici, en dehors de tout support textuel. Effel (1968) défend ainsi une "pasigraphie » universelle (le lien avec l'idéal de « langue parfaite » de Leibniz est explicitement établi dans la préface $)^{45}$.

Dans son Avant projet pour une écriture universelle, l'auteur commence par rassembler des "graphismes, déjà utilisés dans des écritures» (1968:12) - parmi lesquels apparaissent des signes de ponctuation (<?> : «notion d'interroger », <( )> : «notion d'isoler » et <:> «notion d'énoncer»). Il n'est pas question ici d'inventer de nouveaux signes de ponctuation, mais d'utiliser certains signifiants graphiques issus de la ponctuation pour composer d'autres idéogrammes: par exemple, <?> sert à créer, associé à d'autres éléments, un nom (question), un verbe (interroger), des pronoms (que? d'où ?...), un adverbe (peut-être), etc. Utilisé de manière horizontale, le signe exclamatif devient "sursigne » (1968: 33) ; pour composer une interjection, par exemple. Dans les lignes de conclusion, la « portée musicale » apparaît comme un des « aménagements » susceptibles d'améliorer le système de notation (Effel, $1968: 36$ ).

70 Le rôle des signes de ponctuation dans la communication visuelle est ici clairement mis en évidence. Si dessinateurs et publicitaires s'appuient souvent sur les valeurs idéogrammatiques de ponctuants expressifs combinés, l'expérience idéogrammatique envisagée ici consiste à se passer de tout élément verbal ${ }^{46}$. 
Dans Une histoire sans mots (2013), Xu Bing propose un récit composé à partir de pictogrammes (figure 6) - dans lequel les signes de ponctuation apparaissent bien comme des idéogrammes indispensables à la structuration du discours.

\section{$k=$, Mhi, $\rightarrow \rightarrow$.

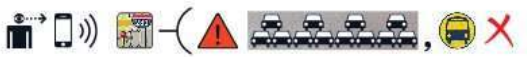

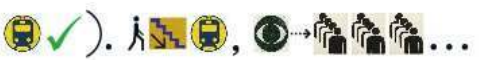

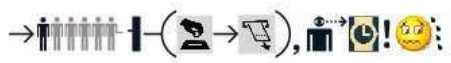

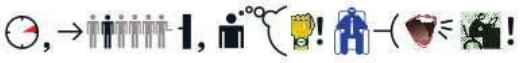

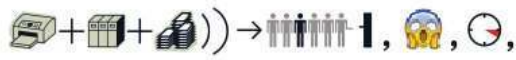

Figure 6 : Extrait de Xu Bing $(2013: 7)^{47}$

\section{En guise de conclusion}

Ce parcours témoigne de la fascination qu'exerce la ponctuation au fil des siècles : elle inspire discours et pratiques innovantes d'ordre divers. Décriée, elle reste envisagée comme un lieu essentiel de perfectionnement de l'écriture; on la rêve surtout moins « ambiguë » - et donc « enrichie » de nouveaux signes. La clarification de l'écrit et l'aide à la lecture sont ainsi présentées comme des enjeux majeurs ; mais notre étude des discours révèle que ce n'est pas pour autant le critère logico-sémantique qui prévaut au moment de justifier l'introduction de nouveaux signes.

Derrière la reconnaissance des rôles très généraux qui sont assignés à la ponctuation ( lever les ambigüités, faciliter la lecture) -, coexistent en réalité diverses manières d'appréhender ces questions et donc de les résoudre. Les fonctions dérivées associées à la ponctuation, lorsqu'elles sont explicitées, renvoient finalement davantage à une conception oralisante que logico-sémantique (même si ces deux niveaux s'articulent); les discours insistent sur le rôle d'une ponctuation facilitant une lecture oralisée, par l'indication de pauses, de nuances intonatives et expressives - qui servent par ailleurs à lever des ambigüités concernant les intentions du scripteur. Insister sur ces caractéristiques revient à reconnaître indirectement que le système traditionnel de ponctuation, bien que jugé insatisfaisant, assurerait bien l'essentiel des fonctions logicosyntaxiques. 
74 Les propositions d'ajout de signes s'inscrivent dans des contextes fort variés dont il convient bien sûr d'apprécier la singularité (en lien avec une époque, des pratiques de lecture et d'écriture spécifiques, un projet plus ou moins ambitieux de réforme, etc.). Mais au-delà des facteurs de variabilité, ce parcours permet de mettre en lumière la récurrence de certains motifs (notamment via des signes réinventés) et d'établir la persistance de représentations communes, à travers les siècles - en lien avec la double orientation (orale et visuelle) des signes de ponctuation.

Les représentations de la ponctuation dont sont porteuses les propositions d'ajout de signes sont ainsi façonnées plus profondément par leur rapport spécifique à un idéal d'écriture ; les nouveaux signes sont envisagés comme des voies de perfectionnement de l'écrit, participant soit d'un système fantasmé de transcription de l'oral, soit davantage d'un système idéal d'écriture figurative - ces deux voies n'étant nullement incompatibles, comme le soulignent les exemples du corpus étudié, où la quête d'expressivité orale peut être recherchée dans une motivation graphique plus grande du système.

76 La ponctuation et les créations innovantes se déclinent ainsi de diverses manières (selon l'autonomie conférée à l'écrit). S'il apparaît dans les discours étudiés que la conception oralisante reste prépondérante, elle n'exclut pas la reconnaissance, à différentes époques, d'une perspective qui valorise aussi une "ponctuation pour l'œil ». Les expériences plus récentes des $\mathrm{XX}-\mathrm{XXI}^{\mathrm{e}}$ siècles, nourries du développement des arts graphiques, permettent bien sûr de mettre davantage en valeur la dimension figurative de la ponctuation (qu'elle soit ensuite présentée ou non, selon les cas, comme étant au service d'une représentation de l'oral). Il importe ainsi de souligner que la recherche générale d'expressivité traverse l'ensemble des propositions et dépasse les clivages entre conceptions oralisante et autonomiste. Idéogrammes ou pictogrammes dérivés de ponctuation répondent d'abord à ce besoin d'expressivité, en s'appuyant sur des signes iconiquement motivés. Espace privilégié de création linguistique, la ponctuation laisse ainsi la voie ouverte à l'expression d'une plus grande subjectivité (dont témoignent les hapax graphiques recensés) - au risque de s'éloigner finalement de l'idéal d'un système dépourvu d'ambigüité.

La variété de cette ponctuation idéalisée, dotée de nouveaux signifiants graphiques, ne fait que rappeler, par les divers projets qui la sous-tendent, la complexité et la richesse inhérente à l'imaginaire d'une ponctuation essentiellement façonnée, au cours de son histoire, par ses rapports à l'oral et à l'écrit.

\section{BIBLIOGRAPHIE}

ADORNO, Th. W., 2004, Mots de l'étranger et autres essais : notes sur la littérature II, Paris, Éd. de la Maison des sciences de l'homme.

ALVAR EZQUERRA, M., 1999, Manual de redacción y estilo, Madrid, Istmo.

ANIS, J., 1981, « Ecrit/oral : discordances, autonomies, transpositions », Etudes de linguistique

appliquée, $\mathrm{n}^{\circ} 42$, Paris, Didier, p. 7-22. 
ANIS, J., 1988, L'Écriture, théories et descriptions, Bruxelles, Boeck-Wesmael.

BADDELEY, S., 2013, « Un microsystème de ponctuation : les notations didascaliennes dans les pièces pédagogiques de Gérard de Vivre, XVIe siècle ", Circé 3, <http://www.revue-circe.uvsq.fr/ un-microsysteme-de-ponctuation-les-notations-didascaliennes-dans-les-pieces-pedagogiquesde-gerard-de-vivre-xvie-siecle/>

BAZIN, H., 1966, Plumons l'oiseau : divertissement, Paris, Grasset.

BING, X., 2013, Une histoire sans mots, Paris, Grasset.

BLAINE, J., 1980, Reprenons la ponctuation à zéro, Ventabren, Nèpe.

BORGES, J.L., 1996, Obras Completas, Barcelona, Emecé.

BRAHM, Alcanter de (pseud. de Bernhardt, M.) [1899], 1996 : L'Ostensoir des ironies, précédé de Le point sur l'ironie, Schoentjes, Pierre (éd.), La Rochelle, Rumeur des âges.

BUTOR, M., 1964, Répertoire II, Paris, Minuit.

BUTOR, M., 1968, Répertoire III, Paris, Minuit.

CABRERA INFANTE, G., 1976, Exorcismos de esti(l)o, Seix Barral, Barcelona.

CASTELLS, A., 2001, Diccionario de Internet : todos los términos utilizados en la RED, Bilbao, Deusto.

CATACH, N., 1980, « La ponctuation », Langue française, 45, Larousse, Paris, p. 16-27.

CATACH, N., 1991, « La ponctuation et l'acquisition de la langue écrite. Norme, système et stratégies », Pratiques, 70, Metz, CRESEF, p. 49-59.

CATACH, N., 1994, La Ponctuation, Paris, PUF.

CHAOUCHE, S., 2000, « Remarques sur le rôle de la ponctuation dans la déclamation théâtrale du XVII ${ }^{\mathrm{e}}$ siècle », dans J. Dürrenmatt (éd.), La Licorne, 52, UFR Langues Littératures de Poitiers, MSHS, p. 83-92.

CLAUSE, L., 2001, « Vers une nouvelle rationalité », Science et Avenir, n647, p. 79.

COHEN, M., PEIGNOT, J., 2005, Histoire et art de l'écriture, Paris, Laffont.

DAMOURETTE, J., 1939, Traité moderne de ponctuation, Paris, Larousse.

DANDOY-MARCHAL DE CASABIANCA, X., 2007, Le Seizième signe, Paris, Éoliennes.

DEFAYS, J.-M., ROSIER, L., TILKIN, F., (éds), 1998, A qui appartient la ponctuation ? Actes du colloque international et interdisciplinaire de Liège (13-15 Mars 1997), Bruxelles, Duculot.

DEMANUELLI, Cl., 1987, Points de repère : approche interlinguistique de la ponctuation français-anglais, Travaux LVIII, CIEREC, Université de Saint-Etienne.

DERRIDA, J., 1967, De la grammatologie, Paris, Minuit.

DRILLON, J., 1991, Traité de la ponctuation française, Paris, Gallimard.

DÜRRENMATT, J., 1998, Bien coupé, mal cousu : de la ponctuation et de la division du texte romantique, Saint-Denis, Presses Universitaires de Vincennes.

EFFEL, J. (pseud. de Lejeune, Fr.), 1968, Avant-projet pour une écriture universelle, Paris, J. Effel.

FETIVEAU, T., s.d., « Andersen », <http://thierryfetiveau.fr/cms/index.php?page=andersen>

fEYdeAu, G., 1995 [1908], Occupe-toi d'Amélie, Paris, Hachette. 
FOURIER, Ch., 2001 [1829], Le nouveau monde industriel et sociétaire ou Invention du procédé d'industrie attrayante et naturelle distribuée en séries passionnées, Dijon, Presses du réel.

Grand Larousse de la Langue Française, 1986, Paris, Larousse, 7 vol.

GREVISSE, M., 1991, Le Bon Usage, Gembloux, Duculot.

GRIMAREST (J.L. le Gallois, sieur de), 1707, Traité du récitatif dans la lecture, dans l'action publique, dans la déclamation et dans le chant, avec un traité des accens, de la quantité et de la ponctuation, Paris, J. Le Fèvre [accès en ligne: http://www.diachronie.be/ponctuation/1707-grimarest/index.html]

GÜIRALDES, R., 1973, Obras Completas, Buenos Aires, Emecé.

HOUDEBINE, A.-M., 2002, « L'imaginaire linguistique : un niveau d'analyse et un point de vue théorique ", dans A.-.M. Houdebine (éd), L'imaginaire linguistique, Paris / Budapest / Torino, L'Harmattan, p. 9-21; « Imaginaire linguistique : parcours théorique et méthodologique » <http://im-ling.voila.net/theorie_Houdebine2.htm>

HUSTON, K., 2014, « Ceci est un point d'ironie », Courrier International 1210, p.46 (<http:// www.courrierinternational.com/article/2014/01/20/ceci-est-un-point-d-ironie>).

JOBARD, J.-B.-A.-M., 1842, Industrie française, rapport sur l'Exposition de 1839, Bruxelles, l'auteur, vol.2.

LARBAUD, V., 1997, Sous l'invocation de Saint Jérôme, Paris, Gallimard.

LARTHOMAS, P., 1995, Le Langage dramatique, Paris, PUF.

LEMAIRE, J., 1998, « Un système de ponctuation original dans l'œuvre d'un dramaturge français du XVI ${ }^{\mathrm{e}}$ siècle », dans J.-M. Defays et al. (éds), À qui appartient la ponctuation ?, Bruxelles, Duculot, p.47-56.

MARTÍNEZ DE SOUSA, J., 1996, Diccionario de Ortografía, Madrid, Paraninfo.

MESCHONNIC, H., 2000, « La ponctuation, graphie du temps et de la voix », dans J. Dürrenmatt (éd), La Licorne, 52, La Ponctuation, UFR Langues Littératures de Poitiers, MSHS, p. 289-293.

MESCHONNIC, H., 2009 [1982], Critique du rythme : anthropologie historique du langage, Lagrasse, Verdier.

NÈVE, F.-X., 1998, « Une quinzaine de signes de ponctuation en plus ? (Réflexions à partir de mon expérience de la langue des signes des sourds) », dans J.M. Defays et al. (éds), A qui appartient la ponctuation ?, Bruxelles, Duculot, p. 337-348.

PARKES, M. B., 1993, Pause and effect : an introduction to the history of punctuation in the West, Berkeley / Los Angeles, University of California Press.

PEIGNOT, J., 1967, De l'écriture à la typographie, Paris, Gallimard.

PEIGNOT, J., 1993, Typoésie, Paris, Imprimerie nationale.

POLO, J., 1990, Manifiesto ortográfico de la lengua española, Madrid, Visor.

PONGE, M., 2007 [2006], La Ponctuation : opposition oralité / scripturalité (domaine hispanique), Doctorat de l'Université Michel de Montaigne - Bordeaux III, Lille, ANRT.

PONGE, M., 2015, « Voix et ponctuation dans le texte littéraire : quels signes pour la voix ? ", dans M. Olmos (éd.), Les Traces de la voix: douze études, Cahiers de l'ERIAC, Université de Rouen, PRUH.

PONGE, M., (à paraître), « La ponctuation : lieu de (re-)motivation iconique », Cahiers de praxématique, 64. 
QUENEAU, R., 1974 [1933], Le chiendent, Folio, Paris, Gallimard.

REBOTIER, J., 2001, Le Dos de la langue : poésie courbe, Paris, Gallimard.

RÉTIF DE LA BRETONNE, N.-E., 1776 [1769], Le Pied de Fanchette, ou le Soulier couleur-de-rose, La Haie. ROUSSEAU, J.-J., 1968 [1781], Essai sur l'origine des langues : où il est parlé de la mélodie et de l'imitation musicale, Bordeaux, Ducros.

RUDOLPH, M.T., 1997, E-mail : courrier Internet, Paris, Micro application.

SAND, G., 1873 [1871], Impressions et souvenirs, Paris : Michel-Lévy frères.

SECRET, J., s.d, « Les signes d'intonation », <http://www.janesecret.net/signes_intonation/>

SZENDY, P., 2013, À coups de points: la ponctuation comme expérience, Paris, Minuit.

TENATENA, P., 2006, « Ortografía y Literatura », AnMal electrónica, 20, <http://

www.anmal.uma.es/numero20/Tena.htm>.

TOURNIER, Cl., 1980, « Histoire des idées sur la ponctuation : des débuts de l'imprimerie à nos jours ", Langue française, 45, Larousse, Paris, p. 28-40.

VALÉRY, P., 1989, Cahiers I (1894-1914), Paris, Gallimard.

VÉDÉNINA, L., 1973, « La transmission par la ponctuation des rapports du code oral avec le code écrit », Langue Française, 19, Phonétique et Phonologie, Larousse, Paris, p. 33-40.

VÉDÉNINA, L., 1989, Pertinence linguistique de la présentation typographique, Paris, Peeters-Salf. WILKER, J., 2002, « La ponctuation chez Mallarmé », dans Cl. Jamain (éd.), La Voix sous le texte : actes du colloque d'Angers 4 et 5 mai 2000, Angers, Presses de l'Université d'Angers, p. 21-29.

\section{NOTES}

1. L'expression est communément utilisée ; voir <http://classes.bnf.fr/dossiecr/si-parol.htm>.

2. A la dizaine de signes généralement cités (point, virgule, point-virgule, deux-points, points de suspension, d'interrogation, d'exclamation, guillemets, tirets, parenthèses), il arrive d'en associer d'autres (crochets, accolades, barre oblique, pied-de-mouche, etc.) et d'inclure, dans une perspective élargie - telle que celle énoncée par Védénina (1973: 34) - « les procédés typographiques pris dans leur ensemble (en y ajoutant le soulignage, l'emploi des caractères, de l'espace blanc entre les signes, etc.) ».

3. Constitué de signes non-alphabétiques, sans correspondants phonémiques (Tournier, 1980).

4. Sur les origines du concept, voir < http://im-ling.voila.net/theorie_Houdebine2.htm> ; l' imaginaire linguistique est associé à l'expression de " normes subjectives ».

5. Cette idée est aussi présente dans l'Emile : «Le meilleur remède pour éviter cette lourdeur étant de perfectionner la ponctuation, qui rendrait dans une certaine mesure la légèreté du langage écrit au langage oral » (cité note 20, ibid).

6. Nous nous limiterons ici au français et à l'espagnol.

7. Ce terme est récurrent. Voir le commentaire de Demanuelli (1987:115); ou Wilmet (1998 : 8) faisant le même constat : «j'ai perçu les déplorations des écrivains relatives à la pauvreté des ressources graphiques mises à leur disposition, incapables de traduire l'infinie variété des mélodies ».

8. Duclos cité par Derrida (1967 : 323, note 46). 
9. Nous devons à Lemaire (1998) notre connaissance des signes de de Vivre dans Fidélités nuptiales (1589). L'image reproduite ici est issue de : <http://www.revue-circe.uvsq.fr/un-microsystemede-ponctuation-les-notations-didascaliennes-dans-les-pieces-pedagogiques-de-gerard-de-vivrexvie-siecle/>.

10. Sur la déclamation théâtrale au XVII ${ }^{\mathrm{e}}$ s., voir Chaouche (2000).

11. J.-L. le Gallois (sieur de Grimarest) dont des extraits du Traité du récitatif (1707) sont commentés par Parkes (1993 : 138) ; pour un accès facilité au texte de 1707 (conforme à l'édition de Le Fèvre \& Ribou) : http://www.diachronie.be/ponctuation/1707-grimarest/index.html

12. Ortografía de 1754 ; voir Parkes (1993: 138, n.86).

13. Voir Dürrenmatt (1998: 75) sur le rôle «clarifiant » de la ponctuation dans le projet de Fourier ; Szendy (2013), pour le lien avec la dimension politique.

14. Voir « The impact of printing: a precarious balance between logical and rhetorical analysis » in Parkes (1993: 87-96).

15. Huston (2014) le mentionne dans un article sur le point d'ironie.

16. Signe en forme d'arc.

17. Signe en forme de U.

18. Cette partie s'appuie sur des éléments développés dans Ponge (2015).

19. En Espagne, dans les ouvrages de la Real Academia Española, la référence à l'oral est redevenue prédominante depuis l'édition de 1999 (vs 1973) - d'après Ponge (2006 : 45-65).

20. Voir Martínez de Sousa (1996) et Alvar Ezquerra (1999).

21. Voir Védénina $(1989,1973)$ et Anis (1981).

22. La proposition de Damourette est aussi commentée par Demanuelli (1987: 82).

23. Grevisse, 1991, § 1059 : «Paul Claudel a préconisé (et employé dans certains de ses ouvrages) une disposition typographique qu'il appelle "pause » et qui consiste à laisser dans certaines phrases de petits intervalles en blanc ».

24. D’après Larbaud (1997 : 229) : «A une certaine époque, Ricardo Güiraldes se plaignait de l'insuffisance et de l'imprécision des signes de ponctuation traditionnels. Il aurait voulu les remplacer ou les compléter par des signes musicaux : soupirs, ou demi-soupirs. Mais il paraît avoir renoncé assez tôt à cette théorie ».

25. D'après Dandoy (2007 : 59).

26. Martínez de Sousa (1996) distingue « signos de entonación » et «signos de puntuación ».

27. Pour d'autres variantes du point d'ironie, voir Huston (2014).

28. Voir Valéry, (1989 [1894-1914] : 474); Peignot (1967: 239); Nève (1998: 347); Cohen \& Peignot (2005 : 2093).

29. D'après l'expression de Meschonnic (2009 [1972] : 300) se référant à la tentative de Pound « d'idéogrammatiser l'écrit occidental ».

30. Son poème «Chœur » s'apparente à une véritable partition musicale (2001 : 165-181)

31. Robetier est aussi compositeur.

32. Cet emploi est mentionné aussi par Larthomas (1995: 54).

33. Voir Ponge (2015 [à paraître]) pour une réflexion sur la «(re)motivation iconique » de la ponctuation; des éléments d'illustration sont repris ici.

34. Signe consistant en une surimpression du point d'exclamation sur le point d'interrogation.

35. La virgule se substitue au point rond de base de l'exclamation. Régulièrement réinventée, elle est défendue avec ferveur par Dandoy.

36. Cabrera Infante (1976: 98) joue de l'homonymie de coma en espagnol (« virgule » ou « coma » en français).

37. Selon la définition d'Anis (1988: 246) : «signe graphique dont la forme procéderait par imitation plus ou moins directe de la réalité extra-linguistique qu'il représente, avec une part variable de figuration et de symbolisme» 
38. Voir http://www.janesecret.net/signes_intonation/

39. Voir http://thierryfetiveau.fr/cms/index.php?page=andersen.

40. Selon Rudolph (1997 : 205).

41. Emoticône de « joie »

42. Emoticône de « tristesse »

43. D'après http://www.franceculture.fr/emission-pixel-emoji-un-langage-universel-2014-12-26 [dernière consultation: 22-01-2015]. La référence à un "langage universel », nommé aussi « esperanto 2.0 », témoigne de la prégnance d'un modèle idéal d'écriture figurative.

44. A la fois extravagant et fort documenté, ce livre se présente comme la réédition de : Le treizième signe ou La nouvelle ponctuation ancienne et moderne, 2003.

45. Dandoy (2007) nous a mis sur la piste d'Effel (1968).

46. Nodier est souvent signalé comme pionnier pour sa page de signes de ponctuation, intitulé « le meilleur du livre » (Moi-même, écrit en 1800, publié en 1921) - en couverture de Licorne, 12, 2000 ; http://classes.bnf.fr/ecritures/arret/signe/ponctuation/04.htm. 47. Image importée de <http://rue89.nouvelobs.com/2013/11/24/saurez-dechiffrer-l-histoiresans-mots-chinois-xu-bing-247672>.

\section{RÉSUMÉS}

Cette étude vise à questionner les représentations dominantes qui façonnent l'imaginaire linguistique de la ponctuation (française et espagnole) à partir d'une analyse des critiques séculaires adressées à son système et des discours qui accompagnent diverses propositions d'ajout de signes. Au-delà de la singularité des projets examinés (entre quête d'un système idéal de notation de l'oral et exploration des potentialités figuratives d'une écriture idéogrammatique, voire pictogrammatique), l'étude révèle la prégnance de certaines représentations en démontrant notamment que les références à l'oral continuent d'habiter la plupart des discours et pratiques innovantes des XX-XXI ${ }^{\mathrm{e}}$ siècles.

This study aims to examine the prevailing representations that mould the "Linguistic Imaginary" (Houdebine) of French and Spanish punctuations: it is based on an analysis of secular criticisms of the punctuation system and the examination of discourses and proposals for adding new punctuation marks. Beyond the singularity of the analysed projects (between the search for an ideal system of transcription and the search for exploration of figurative potentialities: ideographic, pictographic notations), the study shows the ascendency of some representations and demonstrates that oral references are still included in most of discourses and innovative practices of the 20-21th centuries.

\section{INDEX}

Mots-clés : ponctuation à valeur expressive, ponctuation à valeur sémantique, notation de l'oral, idéogrammes pictogrammes 


\section{AUTEUR}

\section{MYRIAM PONGE}

Université Paris 8 / Vincennes - Saint-Denis, LER, EA 1578 